\title{
SPARSITY-BASED DOA ESTIMATION USING CO-PRIME ARRAYS
}

\author{
Yimin D. Zhang ${ }^{\dagger}$, Moeness G. Amin ${ }^{\dagger}$, and Braham Himed ${ }^{\ddagger}$ \\ $\dagger$ Center for Advanced Communications, Villanova University, Villanova, PA 19085, USA \\ $\ddagger$ RF Technology Branch, Air Force Research Lab (AFRL/RYMD), WPAFB, OH 45433, USA
}

\begin{abstract}
In this paper, we propose co-prime arrays for effective direction-of-arrival (DOA) estimation. To fully utilize the virtual aperture achieved in the difference co-array constructed from a co-prime array structure, sparsity-based spatial spectrum estimation technique is exploited. Compared to existing techniques, the proposed technique achieves better utilization of the co-array aperture and thus results in increased degrees-of-freedom as well as improved DOA estimation performance.
\end{abstract}

Index Terms - Co-prime array, sparse array, difference co-array, direction finding, compressive sensing

\section{INTRODUCTION}

Sparse arrays achieve higher degrees-of-freedom (DOFs) by reducing the number of redundant sensors under the co-array equivalence [1]. A minimum redundancy array (MRA) is a well-known example of sparse linear array structures which, for a given number of elements, maximizes the number of consecutive virtual elements in the resulting difference coarray [2]. Minimum hole arrays (also known as the Golomb arrays) minimize the number of holes in the difference coarray [3]. Nested arrays [4], which are obtained by combining two or more uniform linear arrays (ULAs) with ascending inter-element spacing, is another exciting approach for increasing array DOFs.

Recently, the co-prime array has been proposed as an attractive technique for sparse arrays construction [5]. A coprime array utilizes a co-prime pair of uniform linear subarrays, where one is of $M$ sensors with an inter-element spacing of $N$ units, whereas the other is of $N$ elements with an inter-element spacing of $M$ units. The numbers $M$ and $N$ are chosen to be co-prime. The unit inter-element spacing, $d$, is typically set as half wavelength, or $\lambda / 2$. Because the two sub-arrays share the first sensor at the zeroth position, the corresponding co-prime array has a total number of $M+N-1$ sensors and achieves $O(M N)$ DOFs, which is much larger than the number of physical array elements, $M+N-1$. The increased DOFs can be exploited in direction-of-arrival (DOA) estimation and beamforming problems for improved

The work of Y. D. Zhang and M. G. Amin was supported in part by a subcontract with Dynetics, Inc. for research sponsored by the Air Force Research Laboratory (AFRL) under Contract FA8650-08-D-1303, and by the Office of Naval Research (ONR) under grant N00014-13-1-0061. performance and to handle more signals than the number of physical sensors $[5,6]$.

In [6], a new technique is proposed to use co-prime arrays for DOA estimation of uncorrelated sources. This technique recognizes that, by vectorizing the co-prime array covariance matrix, a new formulation involving the steering vectors of a virtual array with an extended aperture can be achieved. The drawback of this formulation, however, is that the secondorder statistics, in particular the source power, now replaces the data sequence. Since source power assumes constant values, the problem becomes DOA estimation of coherent signals or a single snapshot. In this case, subspace-based DOA estimation techniques, such as the commonly used MUSIC algorithm, fail to estimate multiple sources unless source decorrelation is performed. The spatial smoothing technique is applied in [6] to perform signal decorrelation and restore the full rank of the resulting data covariance matrix. The application of spatial smoothing, however, implies significant reduction of the obtained virtual array aperture. As such, the DOFs as well as the performance are compromised.

It is well known that the maximum likelihood (ML) algorithm can resolve the DOAs of multiple sources even when they are coherent. In the presence of multiple sources, however, ML requires a multi-dimensional search and thus is considered impractical due to its prohibitively high complexity. Nevertheless, the recent advances in compressive sensing enable an efficient solution of such ML problems by utilizing the sparsity property of the sources in the spatial domain. DOA estimation can be implemented using a number of techniques $[7,8]$, such as the least absolute shrinkage and selection operator (Lasso), orthogonal matching pursuit (OMP), and compressive sampling matched pursuit (CoSaMP) [9, 10, 11]. The use of sparse support recovery techniques for DOA estimation using nested arrays was considered in $[12,13]$. On the other hand, a parameter-free algorithm, referred to as the iterative adaptive approach (IAA), was proposed recently in [14] and extended in $[15,16,17]$. These techniques are insensitive to practical impairments such as the availability of a few (even single) snapshots and coherent sources.

In this paper, we propose the use of sparse signal recovery methods in the co-prime arrays for effective DOA estimations. As it becomes clear from the above discussion, applying these methods to co-prime array allows us to estimate DOA information from the single snapshot or coherent data samples of the difference co-arrays. In doing so, there is no need to perform spatial smoothing and, as such, the entire virtual array aperture can be fully utilized. The Lasso algorithm 
is considered in this paper for sparse signal recovery, but other methods may also be applied. Different co-prime array structures are compared for DOA estimations.

Notations: We use lower-case (upper-case) bold characters to denote vectors (matrices). In particular, $\mathbf{I}_{N}$ denotes the $N \times N$ identity matrix. (. $)^{T}$ and (.) ${ }^{H}$ respectively denote the transpose and conjugate transpose of a matrix or vector. $\operatorname{vec}(\cdot)$ denotes the vectorization operator that turns a matrix into a vector by stacking all columns on top of the another, and $\operatorname{diag}(\mathbf{x})$ denotes a diagonal matrix that uses the elements of $\mathbf{x}$ as its diagonal elements. $\|\cdot\|_{2}$ denotes the Euclidean $\left(l_{2}\right)$ norm of a vector, whereas $\|\cdot\|_{0}$ and $\|\cdot\|_{1}$ respectively denote the $l_{0}$ and $l_{1}$ norms. $\mathrm{E}(\cdot)$ denotes the statistical expectation operator. $\bigotimes$ denotes the Kronecker product, and real $(\cdot)$ and $\operatorname{imag}(\cdot) \operatorname{represent}$ the real and imaginary part operations.

\section{SYSTEM MODEL}

A co-prime array, as illustrated in Fig. 1, consists of a coprime pair of uniform linear sub-arrays, where one sub-array uses $M$ sensors with an inter-element spacing of $N$ units, whereas the other uses $N$ elements with an inter-element spacing of $M$ units [5]. $M$ and $N$ are chosen to be co-prime and, without loss of generality, we assume $M<N$. The unit inter-element spacing, $d$, is usually set as half wavelength, or $\lambda / 2$.

The resulting $N+M-1$ elements are positioned at

$S=\{M n d, 0 \leq n \leq N-1\} \cup\{N m d, 0 \leq m \leq M-1\}$.

Because the two sub-arrays share the first sensor at the zeroth position, the total number of sensors in the co-prime array is $M+N-1$.

Denote $\mathbf{p}=\left[p_{1}, \ldots, p_{M+N-1}\right]^{T}$ as the positions of the array sensors where $p_{i} \in S, \forall i$, where the first sensor is assumed as the reference, i.e., $p_{1}=0$. Assume that $D$ uncorrelated signals imping on the array from angles $\Theta=\left[\theta_{1}, \ldots, \theta_{D}\right]^{T}$, and their discritized baseband waveforms are expressed as $s_{d}(t), t=1, \ldots, T$, for $d=1, \ldots, D$. Then, the data vector received at the co-prime array is expressed as

$$
\mathbf{x}(t)=\sum_{d=1}^{D} \mathbf{a}\left(\theta_{d}\right) s_{d}(t)+\mathbf{n}(t)=\mathbf{A} \mathbf{s}(t)+\mathbf{n}(t)
$$

where

$$
\mathbf{a}\left(\theta_{d}\right)=\left[1, e^{j \frac{2 \pi p_{2}}{\lambda} \sin \left(\theta_{d}\right)}, \ldots, e^{j \frac{2 \pi p_{M}+N-1}{\lambda} \sin \left(\theta_{d}\right)}\right]^{T}
$$

is the steering vector of the array corresponding to $\theta_{d}, \mathbf{A}=$ $\left[\mathbf{a}\left(\theta_{1}\right), \ldots, \mathbf{a}\left(\theta_{D}\right)\right]$, and $\mathbf{s}(t)=\left[s_{1}(t), \ldots, s_{D}(t)\right]^{T}$. The elements of the noise vector $\mathbf{n}(t)$ are assumed to be independent and identically distributed (i.i.d.) random variables following the complex Gaussian distribution $\mathcal{N} C\left(0, \sigma_{n}^{2}\right)$, i.e., of zero mean and variance $\sigma_{n}^{2}$.

The covariance matrix of data vector $\mathbf{x}(t)$ is obtained as

$$
\begin{aligned}
\mathbf{R}_{\mathbf{x x}} & =\mathbf{E}\left[\mathbf{x}(t) \mathbf{x}^{H}(t)\right]=\mathbf{A} \mathbf{R}_{\mathbf{s s}} \mathbf{A}^{H}+\sigma_{n}^{2} \mathbf{I}_{M+N-1} \\
& =\sum_{d=1}^{D} \sigma_{d}^{2} \mathbf{a}\left(\theta_{d}\right) \mathbf{a}^{H}\left(\theta_{d}\right)+\sigma_{n}^{2} \mathbf{I}_{M+N-1},
\end{aligned}
$$

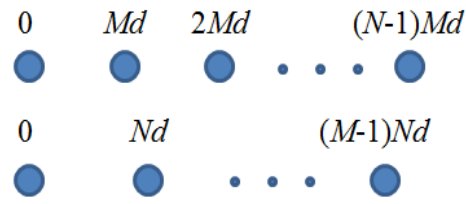

Fig. 1. Co-prime array configuration.

where $\mathbf{R}_{\mathbf{s s}}=\mathrm{E}\left[\mathbf{s}(t) \mathbf{s}^{H}(t)\right]=\operatorname{diag}\left(\left[\sigma_{1}^{2}, \ldots, \sigma_{D}^{2}\right]\right)$ is the source covariance matrix, where $\sigma_{d}^{2}$ denotes the input signal power of the $d$ th source, $d=1, \ldots, D$. In practice, the covariance matrix is replaced by the following sample average:

$$
\hat{\mathbf{R}}_{\mathbf{x x}}=\frac{1}{T} \sum_{t=1}^{T} \mathbf{x}(t) \mathbf{x}^{H}(t) .
$$

\section{CO-PRIME MUSIC ALGORITHM}

In this section, we summarize the concept of co-prime MUSIC algorithm proposed in [6]. The entries of the covariance matrix $\mathbf{R}_{\mathbf{x x}}$ correspond to different lags. From antennas located at the $m$ th and $n$th positions in $\mathbf{p}$, the correlation $\mathrm{E}\left[x_{m}(t) x_{n}^{*}(t)\right]$ yields an entry in $\mathbf{R}_{\mathbf{x x}}$ with lag $p_{m}-p_{n}$. As such, all the available values of $m$ and $n$, where $0 \leq n \leq$ $N-1$ and $0 \leq m \leq M-1$, yields virtual sensors of the following difference co-array:

$$
C_{S}=\{\mathbf{z} \mid \mathbf{z}=\mathbf{u}-\mathbf{v}, \mathbf{u} \in S, \mathbf{v} \in S\} .
$$

Vectorizing $\mathbf{R}_{\mathbf{x x}}$ yields

$$
\mathbf{z}=\operatorname{vec}\left(\mathbf{R}_{\mathbf{x x}}\right)=\tilde{\mathbf{A}} \mathbf{b}+\sigma_{n}^{2} \tilde{\mathbf{i}},
$$

where $\tilde{\mathbf{A}}=\left[\tilde{\mathbf{a}}\left(\theta_{1}\right), \ldots, \tilde{\mathbf{a}}\left(\theta_{D}\right)\right], \tilde{\mathbf{a}}\left(\theta_{d}\right)=\mathbf{a}^{*}\left(\theta_{d}\right) \otimes \mathbf{a}\left(\theta_{d}\right)$, $\mathbf{b}=\left[\sigma_{1}, \ldots, \sigma_{D}\right]^{T}$, and $\tilde{\mathbf{i}}=\operatorname{vec}\left(\mathbf{I}_{M+N-1}\right)$. Assume that the noise variance can be estimated and the effect of $\sigma_{n}^{2} \tilde{\mathbf{i}}$ can be compensated for in the above expression. Then, the vector $\mathbf{z}$ amounts to the received data from a virtual array with a much longer array aperture whose corresponding steering matrix is defined by $\tilde{\mathbf{A}}$. However, the virtual source signal becomes a single snapshot of $\mathbf{b}$. Therefore, the rank of the noise-free covariance matrix of $\mathbf{z}, \mathbf{R}_{\mathbf{z z}}=\mathbf{z z}^{H}$, is one, and subspace-based DOA estimation techniques, such as MUSIC, fail to yield DOA estimates when multiple signals imping to the array. Notice that the use of multiple segments of data may yield multiple samples of $\mathbf{b}$, but in so doing the rank of $\mathbf{R}_{\mathbf{z z}}$ is not increased because these data samples are coherent as $\mathbf{b}$ is constant.

To overcome this problem, as shown in Fig. 2, it is proposed in [6] to double the number of array sensor in the first sub-array, which has fewer sensors than the other sub-array. To distinguish the notation from the original co-prime array structure, we denote the number of sensors in the two subarrays as $N^{\prime}$ and $2 M^{\prime}$. Because the zeroth sensor positions of the two arrays are collocated, the total number of the physical sensors in this co-prime array structure is $N^{\prime}+2 M^{\prime}-1$. 


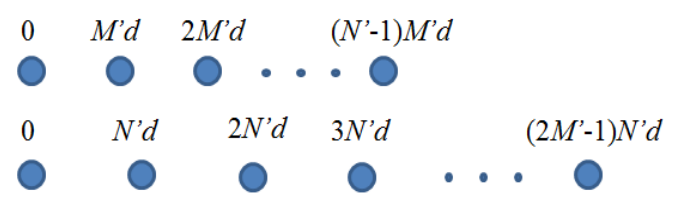

Fig. 2. Alternative co-prime array configuration for DOA estimation.

As such, the lag difference generated by this co-prime array includes all the $2 M^{\prime} N^{\prime}+1$ lag difference from $-M^{\prime} N^{\prime}$ to $M^{\prime} N^{\prime}$. By extracting these $2 M^{\prime} N^{\prime}+1$ lags that correspond to a ULA with antenna positions at $n d$, where $-M^{\prime} N^{\prime} \leq$ $n \leq M^{\prime} N^{\prime}$, we can construct a $\left(2 M^{\prime} N^{\prime}+1\right) \times\left(2 M^{\prime} N^{\prime}+1\right)$ covariance matrix, denoted as $\mathbf{R}_{\mathbf{z z}}^{\prime}$. From the $M^{\prime} N^{\prime}+1$ diagonal sub-matrices, each of size $\left(M^{\prime} N^{\prime}+1\right) \times\left(M^{\prime} N^{\prime}+1\right)$, we can perform spatial smoothing to obtain an $\left(M^{\prime} N^{\prime}+1\right) \times$ $\left(M^{\prime} N^{\prime}+1\right)$ covariance matrix, which has a rank of $M^{\prime} N^{\prime}+1$. As such, the co-prime MUSIC algorithm achieves a DOF of $M^{\prime} N^{\prime}$ from $2 M^{\prime}+N^{\prime}-1$ physical sensors. We observe, however, that the available DOFs are ineffectively used because of the exploitation of spatial smoothing. As a matter of fact, the usable DOFs are only approximately half of the continuous lags.

\section{PROPOSED APPROACH}

We consider solving (7) in terms of the sparse signal recovery through compressive sensing. The desired result of $\mathbf{b}$ is represented as the solution to the following constrained minimization problem

$$
\hat{\mathbf{b}}=\arg \min _{\mathbf{b}}\|\mathbf{b}\|_{0} \quad \text { s.t. } \quad\left\|\mathbf{z}-\tilde{\mathbf{A}} \mathbf{b}-\sigma_{n}^{2} \tilde{\mathbf{i}}\right\|_{2}<\epsilon,
$$

where $\epsilon$ is a user-specific bound. For notational compactness, we define $\mathbf{B}=[\tilde{\mathbf{A}}, \tilde{\mathbf{i}}]$ and $\mathbf{r}=\left[\mathbf{b}^{T}, \sigma_{n}^{2}\right]^{T}=\left[\sigma_{1}^{2}, \ldots, \sigma_{D}^{2}, \sigma_{n}^{2}\right]$. Then, the above expression can be reformulated as

$$
\hat{\mathbf{r}}=\arg \min _{\mathbf{r}}\|\mathbf{r}\|_{0} \quad \text { s.t. } \quad\|\mathbf{z}-\mathbf{B r}\|_{2}<\epsilon .
$$

This type of problems has been the objective of intensive studies in the area of compressive sensing. A number of effective numerical computation methods have been developed. In this paper, we use the batch Lasso method, but other methods may also be used. Lasso is one of the most important techniques for sparse signal recovery which uses the $l_{1}$-norm to enforce sparsity, i.e., the $l_{0}$ norm in the above expression is replaced by the $l_{1}$ norm. By defining matrix the $\mathbf{B}^{\mathbf{g}}$ as the collection of steering vectors over a finite grid $\theta_{1}^{\mathrm{g}}, \ldots, \theta_{K}^{\mathrm{g}}$, where $K \gg D$, the Lasso objective function is defined as

$$
\hat{\mathbf{r}}^{\mathrm{g}}=\arg \min _{\mathbf{r}^{\mathrm{g}}}\left[\frac{1}{2}\left\|\mathbf{z}-\mathbf{B}^{\mathrm{g}} \mathbf{r}^{\mathrm{g}}\right\|_{2}+\lambda_{t}\left\|\mathbf{r}^{\mathrm{g}}\right\|_{1}\right],
$$

where the $l_{2}$ norm in the objective function denotes the ordinary least-squares (OLS) cost function, and the $l_{1}$ norm involves the sparsity constraint. In addition, $\lambda_{t}$ is a penalty parameter which can be tuned to trade off the OLS error for the number of nonzero entries (degree of sparsity) in the estimates [9]. The above Lasso objective is convex in $\mathbf{r}^{\mathrm{g}}$, and can be optimized using linear programming techniques [18]. The last entry of $\mathbf{r}^{\mathrm{g}}$ denotes the estimate of $\sigma_{n}^{2}$, whereas the positions and values of the non-zero entries in the other elements of $\mathbf{r}^{\mathrm{g}}$ represent the DOA estimates and the respective signal power.

The Lasso algorithm is developed to solve real-value problems, whereas $\mathbf{B}^{\mathrm{g}}$ and $\mathbf{z}$ in (10) are generally complex. To modify the problem (10) to a real-valued problem, we notice that $\mathbf{r}^{\mathrm{g}}$ is real-valued, and denote $\tilde{\mathbf{B}}^{\mathrm{g}}=$ $\left[\operatorname{real}\left(\mathbf{B}^{\mathrm{g}}\right)^{T}, \operatorname{imag}\left(\mathbf{B}^{\mathrm{g}}\right)^{T}\right]^{T}$ and $\tilde{\mathbf{z}}=\left[\operatorname{real}(\mathbf{z})^{T}, \operatorname{imag}(\mathbf{z})^{T}\right]^{T}$. Then, the above expression is equivalently described as

$$
\hat{\mathbf{r}}^{\mathrm{g}}=\arg \min _{\mathbf{r}^{\mathrm{g}}}\left[\frac{1}{2}\left\|\tilde{\mathbf{z}}-\tilde{\mathbf{B}}^{\mathrm{g}} \mathbf{r}^{\mathrm{g}}\right\|_{2}+\lambda_{t}\left\|\mathbf{r}^{\mathrm{g}}\right\|_{1}\right] .
$$

We make the following two remarks:

1. Two co-prime array structures are shown in Figs. 1 and 2. Given the same number of the total physical sensors (i.e., $M+N-1=2 M^{\prime}+N^{\prime}-1$, the second array geometry tends to achieve a larger number of consecutive virtual sensors and thus generally yields a better DOA estimation performance.

2. The Lasso-based spectrum estimation technique may induce spurious peaks by undesirable estimates of $\mathbf{r}$, especially in low signal-to-noise ratio (SNR) situations. When the number of sources is smaller than the number of physical array sensors, the number of sources can be estimated according to the Akaike information criterion (AIC) or minimum description length (MDL) criterion [19]. In this case, the DOA estimates can be empirically obtained by selecting $D$ dominant peaks of the spatial spectrum. When the number of sources is larger than the number of physical array sensors, however, then the number of sources cannot be estimated in this manner.

\section{SIMULATION RESULTS}

We consider co-prime arrays consisting of 10 physical antenna sensors. For the first co-prime array geometry, we have $N=6$ and $M=5$, The sensor positions of the two subarrays are respectively $[0,6,12,18,24] d$ and $[0,5,10,15$, $20,25] d$, where the zeroth position is shared by both subarrays, and the unit inter-element spacing $d$ is chosen to be half wavelength. On the other hand, the second co-prime array geometry can be designed by taking $N^{\prime}=5$, and $M^{\prime}=3$, where the sensor are respectively located at $[0,3,6,9,12] d$ and $[0,5,10,15,20,25] d$. Both arrays have a maximum virtual array lag of $25 d$. However, the first co-prime array geometry achieves consecutive virtual array lags between $-10 d$ and $10 d$, whereas the second one achieves consecutive lags between $-17 d$ and $17 d$. The number of unique lag positions is 39 for the first co-prime array and 43 for the second one.

We consider 17 narrowband sources uniformly distributed between $-50^{\circ}$ and $50^{\circ}$. This is the maximum number of 
sources the co-prime MUSIC algorithm can resolve. The input SNRs for all sources are assumed to be identical. The number of snapshots is 500 .

The estimated spectrum from the Lasso method is shown in Figs. 3 and 4 respectively for the two co-prime array structures, where the input SNR levels of $-10 \mathrm{~dB}$ and $0 \mathrm{~dB}$ are considered. $\lambda_{t}=0.25$ is chosen to perform the Lasso method. It is seen that, while all the 17 sources are correctly or closely identified in both co-prime array geometries, spurious peaks are regularly observed around $-9^{\circ}$ and $9^{\circ}$ in the first co-prime array. For comparison, the results obtained from the co-prime MUSIC is shown in Fig. 5. It is clear that the co-prime MUSIC is unable to correctly identify the true source spectra, particularly when the input SNR is low.

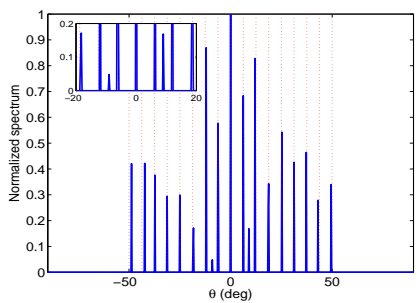

(a) input $\mathrm{SNR}=-10 \mathrm{~dB}$

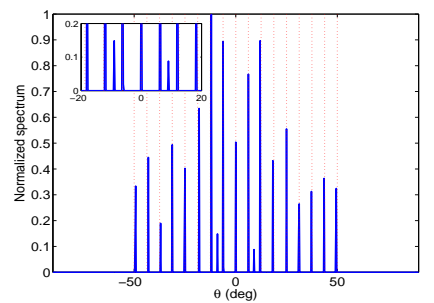

(b) input $\mathrm{SNR}=0 \mathrm{~dB}$
Fig. 3. Spatial spectrum estimated using the Lasso method (first co-prime array geometry).

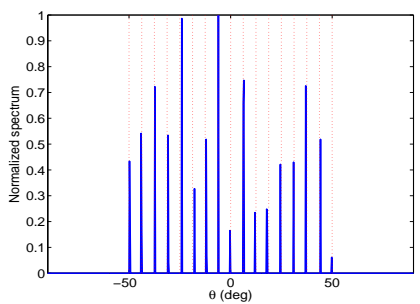

(a) input $\mathrm{SNR}=-10 \mathrm{~dB}$

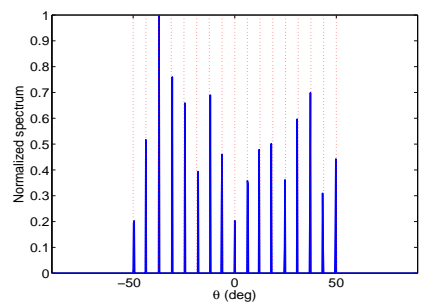

(b) input $\mathrm{SNR}=0 \mathrm{~dB}$
Fig. 4. Spatial spectrum estimated using the Lasso method (second co-prime array geometry).

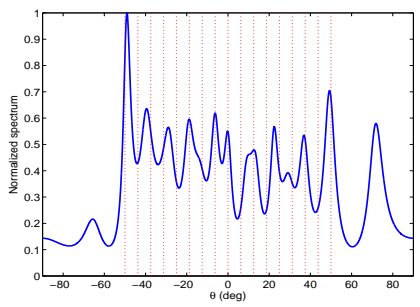

(a) input $\mathrm{SNR}=-10 \mathrm{~dB}$

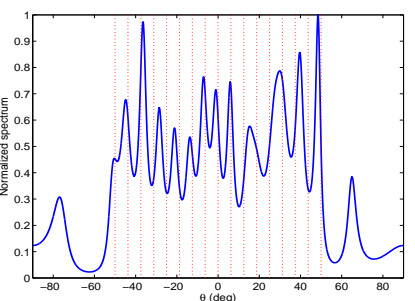

(b) input $\mathrm{SNR}=0 \mathrm{~dB}$
Fig. 5. Spatial spectrum estimated using the co-prime MUSIC method.

\section{CONCLUSIONS}

We have proposed a sparse signal recovery technique for DOA estimation in co-prime arrays. For co-prime arrays, a single snapshot condition arises in the process of formulating the virtual aperture with extended steering vectors. The Lasso algorithm is used to estimate the spatial signal spectrum based on the difference co-array with a single snapshot. The proposed technique achieves higher degrees-of-freedom than the existing co-prime MUSIC. The superior performance of the proposed technique is verified using simulation results.

\section{REFERENCES}

[1] R. T. Hoctor and S. A. Kassam, "The unifying role of the coarray in aperture synthesis for coherent and incoherent imaging," Proc. IEEE, vol. 78, no. 4, pp. 735-752, April 1990.

[2] A. Moffet, "Minimum-redundancy linear arrays," IEEE Trans. Antennas Propagat., vol. 16, no. 2, pp. 172-175, March 1968.

[3] G. S. Bloom and S. W. Golomb, "Application of numbered undirected graphs," Proc. IEEE, vol. 65, no. 4, pp. 562-570, April 1977.

[4] P. Pal and P. P. Vaidyanathan, "Nested Arrays: A novel approach to array processing with enhanced degrees of freedom," IEEE Trans. Signal Proc., vol. 58, no. 8, pp. 4167-4181, Aug. 2010.

[5] P. P. Vaidyanathan and P. Pal, "Sparse sensing with co-prime samplers and arrays," IEEE Trans. Signal Proc., vol. 59, no. 2, pp. 573-586, Feb. 2011.

[6] P. Pal and P. P. Vaidyanathan, "Coprime sampling and the MUSIC algorithm," in Proc. IEEE Digital Signal Proc. Workshop and IEEE Signal Proc. Education Workshop, Sedona, AZ, Jan. 2011.

[7] A. Panahi and M. Viberg, "On the resolution of the LASSObased DOA estimation method," in Proc. Int. ITG Workshop on Smart Antennas, Aachen, Germany, Feb. 2011.

[8] N. Hu, D. Xu, X. Xu, and Z. Ye, "Wideband DOA estimation from the sparse recovery perspective for the spatial-only modeling of array data," Signal Proc., vol. 92, pp. 1359-1364, 2012.

[9] R. Tibshirani, "Regression shrinkage and selection via the lasso," J. Royal Statistical Society, Series B, vol. 58, no. 1, pp. 267-288, 1996.

[10] J. A. Tropp and A. C. Gilbert, "Signal recovery from random measurements via orthogonal matching pursuit," IEEE Trans. Info. Theory, vol. 53, no. 12, pp. 4655-4666, 2007.

[11] D. Needella and J. A. Troppb, "CoSaMP: iterative signal recovery from incomplete and inaccurate samples," Applied and Computational Harmonic Analysis, vol. 26, no. 3, pp. 301-321, May 2009.

[12] P. Pal and P. P. Vaidyanathan, "Correlation aware techniques for sparse support recovery," in Proc. IEEE Statistical Signal Processing Workshop, Ann Arbor, MI, pp. 53-56, Aug. 2012.

[13] P. Pal and P. P. Vaidyanathan, "On application of LASSO for sparse support recovery with imperfect correlation awareness," in Proc. Asilomar Conf. Signals, Systems and Computers, Pacific Grove, CA, Nov. 2012.

[14] T. Yardibi, J. Li, P. Stoica, and A. B. Baggeroer, "Source localization and sensing: a nonparameteric iterative adaptive approach based on weighted least squares," IEEE Trans. Aerosp. Electron. Syst., vol. 46, no. 1, pp. 425-443, Jan. 2010. 
[15] W. Roberts, P. Stoica, J. Li, T. Yardibi, and F. A. Sadjadi, "Iterative adaptive approaches to MIMO radar imaging," IEEE J. Selected Topics Signal Proc., vol. 4, no. 1, pp. 5-20, Feb. 2010.

[16] P. Stoica, P. Babu, and J. Li, "New method of sparse parameter estimation in separable models and its use for spectral analysis of irregularly sampled data," IEEE Trans. Signal Proc., vol. 59, no. 1, pp. 35-47, Jan.- 2011.

[17] P. Stoica, P. Babu, and J. Li, "SPICE: A sparse covariancebased estimation method for array processing," IEEE Trans. Signal Proc., vol. 59, no. 2, pp. 629-638, Feb. 2011.

[18] D. P. Bertsekas, Nonlinear Programming, Second Edition. Athena Scientific, 1999.

[19] M. Wax and T. Kailath, "Detection of signals by information theoretic criteria," IEEE Trans. Acoustics, Speech, Signal Proc., vol. 33, no. 4, pp. 387-392, April 1985. 